Pacific Journal of Mathematics

STOCHASTIC DIFFUSION ON AN UNBOUNDED DOMAIN 


\section{STOCHASTIC DIFFUSION ON AN UNBOUNDED DOMAIN}

\section{ROBERT MARCUS}

In this paper we study a stochastic partial equation of the following form.

$$
\frac{\partial u}{\partial t}=1 / 2 \frac{\partial^{2} u}{\partial x^{2}}-f(u)+\alpha(x, t)
$$

where $f$ is a monotone nonlinear operator and $\alpha$ is a "white noise" process in $x$ and $t$. In a previous paper we demonstrated the existence of a unique solution in a generalized sense for $x$ in a bounded domain. This solution was decomposed into the sum of a stationary process and a transient process. An explicit representation was found for the stationary distribution of the stationary process. If $f$ is an ordinary function of $u(x)$ then the stationary distribution is associated with a Markov process in $x$. The purpose of this paper is to remove the restriction of boundedness for the bounded domain.

The motivation for this study was to establish a link between stochastic partial differential equations and constructive quantum field theory. The basic idea is that the stationary distributions of certain stochastic partial differential equations will be Euclidean Markov fields. See Nelson [3]. For an example see Appendix.

1. Definitions. The equation studied is formally

$$
\begin{gathered}
u_{t}(x, t)=\frac{1}{2} u_{x x}(x, t)-\lambda^{2} u(x, t)-f(u(x, t))+\alpha(x, t) \\
(x \varepsilon(-\infty,+\infty), \lambda>0)
\end{gathered}
$$

and for convenience $u(x, 0)=0, \alpha(x, t)$ is a "white noise" process i.e., $E(\alpha(x, t) \alpha(y, s))=\delta(x-y) \delta(t-s)$.

Converting (1) to an integral equation

$$
u(x, t)=-\int_{0}^{t} \int_{-\infty}^{+\infty} G_{\lambda}(t-s, x, y) f(u(y, s)) d y d s+W(x, t)
$$

with

$$
G_{\lambda}(t-s, x, y)=\exp \left(-\lambda^{2} t-(x-y)^{2} / 2(t-s)\right) / \sqrt{2 \pi(t-s)} .
$$

$W(x, t)$ is a Gaussian process with mean 0 and covariance equal to

$$
E(W(x, t) W(y, s))=\int_{0}^{\min (t, s)} G_{\lambda}(t+s-2 r, x, y) d r
$$

formally 


$$
W(x, t)=\int_{0}^{t} \int_{-\infty}^{+\infty} G_{\lambda}(t-s, x, y) \alpha(y, s) d y d s .
$$

In addition the following conditions are required on $f: R^{1} \rightarrow R^{1}$ :

(i) $(f(u)-f(v))(u-v)>c_{1}|u-v|^{p}$

(ii) $|f(u)|^{q}<c_{2}\left(|u|^{p}+1\right)$

with $c_{1}, c_{2}>0, p>2$, and $q=p /(p-1)$.

Definitions. Let $L_{k}^{p}$ be the Banach space with norm $|\cdot|_{\text {olc }}$ defined by

$$
|u(x)|_{o k}^{p}=\int_{0}^{\infty} \exp (-k|x|)|u(x)|_{d \tau}^{p} \text { with } 0<k / 2<\lambda .
$$

Let $L_{k}^{q}$ be the dual space with norm $|\cdot|_{0 k}^{*}$.

Let $B_{k}$ be the Banach space with norm $|\cdot|_{k}$ satisfying $|u|_{k}^{p}=\int_{0}^{t}|u|_{o k}^{p} d t$. Let $B_{k}^{*}$ be the dual of $B_{k}$ with norm $|\cdot|_{k}^{*}$.

Lemma 1. $W(x, t) \in B_{k}$ almost surely.

$$
\text { Proof. } \begin{aligned}
E\left(W^{2}(x, t)\right) & =\int_{0}^{t} G_{\lambda}(2 t-2 r, x, x) d r \\
& =\int_{0}^{t} \exp \left(-2 \lambda^{2} r\right) / \sqrt{4 \pi r} d r \\
& <\int_{0}^{\infty} \exp \left(-2 \lambda^{2} r\right) / \sqrt{4 \pi r} d r<\infty .
\end{aligned}
$$

From the Gaussian properties of $W$ it follows that

$$
\begin{aligned}
& E\left(\int_{-\infty}^{+\infty} \exp (-k|x|)|W(x, t)|^{p} d x\right)<\infty \\
& \quad \text { uniformly in } t \text { and hence } E\left(|W|_{k}^{p}\right)<\infty .
\end{aligned}
$$

Then Chebyshev's inequality can be used to complete the proof of the lemma.

The method of solving (2) will be to construct a sequence of approximations $u_{N}(x, t)$ that converge to a solution. Let $G_{\lambda N}(t, x, y)$ satisfy

$$
\frac{\partial G_{\lambda N}}{\partial t}=\frac{1}{2} \frac{\partial^{2} G_{\lambda N}}{\partial x^{2}}-\lambda^{2} G_{\lambda N}
$$

$G_{\lambda_{N}}(t, x, y)=0$ for $|x| \geqq N$ or $|y| \geqq N$ and $G_{\lambda_{N}}(0, x, y)=\delta(x-y)$. Using the reflection method it is easy to show that

$$
0 \leqq G_{\lambda}(t, x, y)-G_{\lambda N}(t, x, y) \leqq G_{\lambda}(t, x, 2 N-y)+G_{\lambda}(t, x,-2 N-y) \text {. }
$$


Then $u_{N}(x, t)$ will be the solutions of

$$
u_{N}(x, t)=-\int_{0}^{t} \int_{-N}^{+N} G_{\lambda_{N}}(t-s, x, y) f\left(u_{N}(y, s)\right) d y d s+W(x, t) .
$$

\section{Results.}

THEOREM 1. Equation (3) has a unique solution $u_{N}$ for each $N$ almost surely satisfying $\left|u_{N}\right|_{o k} \leqq c$ where $c$ is independent of $N$.

Proof. This theorem follows from Theorem 1 of Marcus [2] and an estimate similar to Theorem 26.6 of Vainberg [5].

The results of Marcus [2] are not applicable to equation (2) because in general $u \in B_{k}$ does not imply that

$$
\int_{0}^{t} \int_{-\infty}^{+\infty} G_{\lambda}(t-s, x, y) f(u(y, s)) d y d s
$$

is in $B_{k}$. However it will be shown by a series of lemmas that the solutions of (3) converge in $B_{k}$ as $N \rightarrow \infty$ to a solution of (2).

Let

$$
u_{N}(x, t)=-\int_{0}^{t} \int_{-N}^{+N} G_{\lambda N}(t-s, x, y) f\left(u_{N}(y, s)\right) d y d s+W(x, t)
$$

and

$$
u_{M}(x, t)=-\int_{0}^{t} \int_{-M}^{+M} G_{\lambda M}(t-s, x, y) f\left(u_{M}(y, s)\right) d y d s+W(x, t)
$$

with $M>N$.

(4) $u_{N}-u_{M}=-\int_{0}^{t} \int_{-N}^{+N}\left(G_{\lambda N}(t-s, x, y)\left(f\left(u_{N}\right)-f\left(u_{M}\right)\right) d y d s\right.$

$$
\begin{aligned}
& -\int_{0}^{t} \int_{-N}^{+N}\left(G_{\lambda_{N}} f\left(u_{M}\right)-G_{\lambda M} f\left(u_{M}\right)\right) d y d s-\int_{0}^{t} \int_{+N}^{+M T} G_{\lambda M}(t-s, x, y) f\left(u_{M}\right) d y d s \\
& -\int_{0}^{t} \int_{-M}^{-N} G_{\lambda_{M}}(t-s, x, y) f\left(u_{M}\right) d y d s .
\end{aligned}
$$

Multiplying by $\exp (-k|x|)\left(f\left(u_{N}\right)-f\left(u_{M}\right)\right)$ and then integrating

$$
\begin{gathered}
\int_{0}^{T} \int_{-\infty}^{+\infty} \exp (-k|x|)\left(f\left(u_{N}\right)-f\left(u_{M}\right)\right)\left(u_{N}-u_{M}\right) d x d t \\
=\int_{0}^{T} \int_{-\infty}^{+\infty} \exp (-k|x|)\left(f\left(u_{N}\right)-f\left(u_{M}\right)\right)
\end{gathered}
$$

$\times$ [Right hand side of (4)] $d x d t$.

LeMma 2. Left hand side of $(5) \geqq c\left|u_{N}-u_{M}\right|_{k}^{p}$ for some $c>0$. 
Proof.

$$
\begin{aligned}
\int_{0}^{t} \int_{-\infty}^{+\infty} \exp (-k|x|)\left(f\left(u_{N}\right)-f\left(u_{M}\right)\right)\left(u_{N}-u_{M}\right) d x d t \\
\quad \geqq c \int_{0}^{T} \int_{-\infty}^{+\infty} \exp (-k|x|)\left|u_{N}-u_{M}\right|^{p} d x d t=c\left|u_{N}-u_{M}\right|_{k}^{p} .
\end{aligned}
$$

LEMMA 3. Expand the expression on the right hand side of (5) the first term is nonpositive, i.e.,

$$
\begin{gathered}
\int_{0}^{T} \int_{-\infty}^{+\infty} \exp (-k|x|)\left(f\left(u_{N}\right)-f\left(u_{M}\right)\right)\left(\int _ { 0 } ^ { t } \int _ { - N } ^ { + N } G _ { \lambda _ { N } } ( t - s , x , y ) \left(f \left(u_{N}(y, s)\right.\right.\right. \\
\left.\left.-f\left(u_{M}(y, s)\right)\right) d y d s\right) d x d t \leqq 0
\end{gathered}
$$

\section{Proof. Let}

$$
V(x, t)=\int_{0}^{t} \int_{-N}^{+N} G_{\lambda N}(t-s, x, y)\left(f\left(u_{N}(y, s)\right)-f\left(u_{M}(y, s)\right)\right) d y d s .
$$

Then $V_{t}=(1 / 2) V_{x x}-\lambda^{2} V+f\left(u_{N}(x, t)\right)-f\left(u_{M}(x, t)\right)$ with $V(x, t)=0$ if $|x| \geqq N$ and $V(x, 0)=0$. Rewriting the left hand side of (6) using $V$ and then integrating by parts, the left hand side of (6) is equal to

$$
\begin{aligned}
- & \int_{0}^{T} \int_{-\infty}^{+\infty} \exp (-k|x|)\left(V_{t}-\frac{1}{2} V_{x x}+\lambda^{2} V\right) V(x, t) d x d t \\
= & -\frac{1}{2} \int_{-N}^{+N} \exp (-k|x|) V^{2}(x, T) d x-\lambda^{2} \int_{0}^{T} \int_{-N}^{+N} \exp (-k|x|) V^{2}(x, t) d x d t \\
& -\frac{1}{2} \int_{0}^{T} \int_{-N}^{+N} \exp (-k|x|) V_{x}^{2} d x d t-\frac{1}{2} k \int_{0}^{T} V^{2}(0, t) d t \\
& +k^{2} / 4 \int_{0}^{T} \int_{-N}^{+N} \exp (-k|x|) V^{2}(x, t) d x d t \leqq 0
\end{aligned}
$$

since $k / 2<\lambda$ by definition.

To complete the proof that $\lim _{M, N \rightarrow \infty}\left|u_{N}-u_{M}\right|_{k}=0$ it is necessary to show that the remaining three terms on the right hand side go to 0 as $N, M \rightarrow \infty$. The proofs are very similar and therefore only one will be shown in detail.

\section{LeMMA 4. Almost surely}

$$
\begin{aligned}
\lim _{M, N \rightarrow \infty} \int_{0}^{T} \int_{-\infty}^{+\infty} \exp (-k|x|)\left(f\left(u_{N}\right)-f\left(u_{M}\right)\right) \\
\quad \times\left(\int_{0}^{t} \int_{N}^{M} G_{\lambda M}(t-s, x, y) f\left(u_{M}\right) d y d s\right) d x d t=0 .
\end{aligned}
$$


Proof. By repeated use of Hölder's inequality, Theorem 1 and (ii)

$$
\begin{aligned}
& \int_{0}^{T} \int_{-\infty}^{+\infty} \exp (-k|x|)\left(f\left(u_{N}\right)-f\left(u_{M}\right)\right)\left(\int_{0}^{t} \int_{N}^{M} G_{\lambda_{M}}(t-s, x, y) f\left(u_{M}\right) d y d s\right) d x d t \\
& \leqq\left(\int_{0}^{T} \int_{-\infty}^{+\infty} \exp (-k|x|)\left|f\left(u_{N}\right)-f\left(u_{M}\right)\right|^{q} d x d t\right)^{1 / q} \\
& \cdot\left(\int_{0}^{T} \int_{-\infty}^{+\infty} \exp (-k|x|)\left(\int_{0}^{t} \int_{N}^{M} G_{\lambda . M}(t-s, x, y) f\left(u_{M}\right) d y d s\right)^{p} d x d t\right)^{1 / p} \\
& \leqq\left(\int_{0}^{T} \int_{-\infty}^{+\infty} \exp (-k|x|)\left(\left|u_{N}\right|^{p}+\left|u_{M}\right|^{p}\right) d x d t\right)^{1 / q} \\
& \cdot\left(\int _ { 0 } ^ { T } \int _ { - \infty } ^ { + \infty } \operatorname { e x p } ( - k | x | ) \left[\int_{0}^{t}\left[\int_{N}^{M} G_{\lambda, M}^{p}(t-s, x, y) \exp \left(p k_{1}|y|\right) d y\right]^{1 / p}\right.\right. \\
& \left.\left.\times\left[\int_{N}^{M}\left|f\left(u_{M}\right)\right|^{q} \exp \left(-q k_{1}|y|\right)\right]^{1 / q} d s\right]^{p} d x d t\right)^{1 / p} \text {. }
\end{aligned}
$$

[If $k_{1}$ is chosen so that $k>p k_{1}>0$ then almost surely]

$$
\begin{aligned}
& \leqq c_{1}\left(\int_{0}^{T} \int_{-\infty}^{+\infty} \exp (-k|x|)\right. \\
&\left.\times\left[\int_{0}^{t}\left(\int_{N}^{M I} G_{\lambda, I}^{p}(t-s, x, y) \exp \left(p k_{1}|y|\right) d y\right)^{1 / p} d s\right]^{p} d x d t\right)^{1 / p} \\
& \leqq c_{2}\left(\int_{0}^{T} \int_{-\infty}^{+\infty} \exp (-k|x|)\right. \\
& \times\left[\int _ { 0 } ^ { t } \left(\int_{N}^{M} \exp \left(-p \lambda^{2}(t-s)-p(x-y)^{2} / 2(t-s)\right) /(2 \pi(t-s))^{p / 2}\right.\right. \\
&\left.\left.\left.\times \exp \left(p k_{1} y\right) d y\right)^{1 / p} d s\right]^{p} d x d t\right)^{1 / p} \\
& \leqq c_{3}\left(\int_{0}^{T} \int_{-\infty}^{+\infty} \exp (-k|x|)\right. \\
& \times\left[\int _ { 0 } ^ { t } \operatorname { e x p } ( - p \lambda ^ { 2 } ( t - s ) ) \left[\int_{N-x}^{\infty} \exp \left(-p z^{2} / 2(t-s)\right) /(2 \pi(t-s))^{p / 2}\right.\right. \\
&\left.\left.\left.\times \exp \left(p k_{1}(z+x)\right) d z\right]^{1 / p} d s\right]^{p} d x d t\right)^{1 / p} \\
& \leqq c_{4} \\
&\left(\int _ { 0 } ^ { T } \int _ { - \infty } ^ { + \infty } \operatorname { e x p } ( - k | x | + p k _ { 1 } x ) \left[\int_{0}^{t} \exp \left(-p \lambda^{2}(t-s)\right)\right.\right. \\
& \times\left[\sup _{z>N-x} \exp \left(-p z^{2} / 4(t-s)\right) /(2 \pi(t-s))^{p / 4}\right]^{1 / p} \\
&\left.\left.\cdot\left(\int_{N-x}^{\infty} \exp \left(-p z^{2} / 4(t-s)\right) /\left(2 \pi(t-s)^{p / 4} \exp \left(p k_{1} z\right) d z\right)^{1 / p}\right) d s\right]^{p} d x d t\right)^{1 / p} \\
& \leqq c_{5}\left(\int _ { 0 } ^ { T } \int _ { - \infty } ^ { + \infty } \operatorname { e x p } ( - k | x | + p k _ { 1 } x ) \left[\int_{0}^{t} \exp \left(-p \lambda^{2}(t-s)\right)\right.\right. \\
& \quad \times\left[\sup _{z>N-x} \exp \left(-z^{2} / 4(t-s)\right) /(2 \pi(t-s))^{1 / 4}\right]^{p} \\
&\left.\left.\quad \cdot\left[\exp \left((t-s) p k_{1}^{2}\right) /(2 \pi(t-s))^{(p-2) / 4}\right]^{1 / p} d s\right]^{p} d x d t\right)^{1 / p}
\end{aligned}
$$




$$
\begin{aligned}
\leqq & c_{6}\left(\int _ { 0 } ^ { T } \int _ { - \infty } ^ { + \infty } \operatorname { e x p } ( - k | x | + p k _ { 1 } x ) \left[\int_{0}^{t} \exp \left(-p \lambda^{2}(t-s)+(t-s) k_{1}^{2}\right)\right.\right. \\
& \left.\left./(2 \pi(t-s))^{(p-1) / 4 p} \cdot \sup _{z>N-x} \exp \left(-z^{2} / 4(t-s)\right) d s\right]^{p} d x d t\right)^{1 / p} \\
\leqq & c_{7}\left(\int _ { 0 } ^ { T } \int _ { - \infty } ^ { + \infty } \operatorname { e x p } ( - k | x | + p k _ { 1 } x ) \left[\int_{0}^{t} \exp \left(-p \lambda^{2}(t-s)+(t-s) k_{1}^{2}\right)\right.\right. \\
& \left.\left./(2 \pi(t-s))^{(p-1) / 4 p} d s\right]^{p} \cdot\left[\sup _{z>N-x}\left(\exp \left(-p z^{2} / 4 t\right)\right)\right] d x d t\right)^{1 / p} \\
\leqq & c_{8}\left(\int_{0}^{T} \int_{-\infty}^{+\infty} \exp \left(-k|x|+p k_{1} x\right) \sup _{z>N-x}\left(\exp \left(-p z^{2} / 4 t\right)\right) d x d t\right)^{1 / p}
\end{aligned}
$$

where $c_{8}$ depends on $T$ and $W$.

Since $\sup _{z>N-x}\left(\exp \left(-p z^{2} / 4 t\right)\right) \leqq 1$ and $k>p k_{1}$ the Lebesgue dominated convergence theorem can be used to show

$$
\begin{aligned}
\lim _{N \rightarrow \infty} \int_{0}^{T} \int_{-\infty}^{+\infty} \exp \left(-k|x|+p k_{1} x\right) \sup _{z>N-x}\left(\exp \left(-p z^{2} / 4 t\right)\right) d x d t \\
\quad=\int_{0}^{T} \int_{-\infty}^{+\infty} \exp \left(-k \mid x+p k_{1} x\right) \lim _{N \rightarrow \infty} \sup _{z>N-x}\left(\exp \left(-p z^{2} / 4 t\right)\right) d x d t=0 .
\end{aligned}
$$

LeMma 5. Almost surely

$$
\begin{aligned}
\lim _{M, N \rightarrow \infty} \int_{0}^{T} \int_{-\infty}^{-\infty} \exp (-k|x|)\left(f\left(u_{N}\right)-f\left(u_{M}\right)\right) \\
\quad \times\left(\int_{0}^{t} \int_{-M}^{-N} G_{\lambda M}(t-s, x, y) f\left(u_{M}\right) d y d s\right) d x d t=0 .
\end{aligned}
$$

Proof. The proof is almost identical to Lemma 4.

Lemma 6. Almost surely

$$
\begin{aligned}
& \lim _{M, N \rightarrow \infty} \int_{0}^{T} \int_{-\infty}^{-\infty} \exp (-k|x|)\left(f\left(u_{N}\right)-f\left(u_{M}\right)\right) \\
& \quad \times\left(\int_{0}^{t} \int_{-N}^{+N}\left(G_{\lambda, H}(t-s, x, y)-G_{\lambda, M}(t-s, x, y)\right) f\left(u_{M}\right) d y d s\right) d x d t=0 .
\end{aligned}
$$

Proof. The proof is similar to that of Lemma 4. However the estimate $G_{\lambda N}(t-s, x, y)-G_{\lambda M}(t-s, x, y) \leqq G_{\lambda}(t-s, x, 2 N-y)+$ $G_{\lambda}(t-s, x,-2 N-y)+G_{\lambda}(t-s, x, 2 M-y)+G_{\lambda}(t-s, x,-2 M-y)$ from the reflection method is used to complete the proof.

THEOREM 2. $\operatorname{Lim}_{N \rightarrow \infty} u_{N}$ exists in $B_{k}$ almost surely. Also if $u \equiv \lim _{N \rightarrow \infty} u_{N}$ then $|u(\cdot, t)|_{\text {ok }}<c$ almost surely for almost all $t$.

Proof. From (5) using Lemmas 2, 3, 4, 5,6 $\left|u_{N}-u_{M}\right|_{k}^{p}$ is less than or equal to the sum of expressions whose limit as $N, M \rightarrow \infty$ 
is 0 . Hence $u_{N}$ is a Cauchy sequence in $B_{k}$. Since $\left|u_{N}(\cdot, t)\right|_{o k}<c$ almost surely by Theorem 1 the same bound applies to $u$ for almost all $t$.

LeMma 7. $u(x, t)=-\int_{0}^{t} \int_{-\infty}^{+\infty} G_{\lambda}(t-s, x, y) f(u) d y d s+W(x, t)$.

Proof. Since $u_{N}=-\int_{0}^{t} \int_{-N}^{+N} G_{\lambda_{N}}(t-s, x, y) f\left(u_{N}\right) d y d s+W(x, t)$ it is only necessary to show that

$$
\lim _{N \rightarrow \infty}\left|\int_{0}^{t} \int_{-N}^{+N} G_{\lambda N}(t-s, x, y) f\left(u_{N}\right) d y d s-\int_{0}^{t} \int_{-\infty}^{+\infty} G_{\lambda}(t-s, x, y) f(u) d y d s\right|_{p}=0 .
$$

$$
\begin{aligned}
& \int_{0}^{t} \int_{-N}^{+N} G_{\lambda N}(t-s, x, y) f\left(u_{N}\right) d y d s-\int_{0}^{t} \int_{-\infty}^{+\infty} G_{\lambda}(t-s, x, y) f(u) d y d s \\
& \quad=\int_{0}^{t} \int_{-N}^{+N} G_{\lambda_{N}}(t-s, x, y)\left(f\left(u_{N}\right)-f(u)\right) d y d s \\
& \quad+\int_{0}^{t} \int_{-N}^{+N}\left(G_{\lambda_{N}}(t-s, x, y)-G_{\lambda}(t-s, x, y)\right) f(u) d y d s \\
& \quad-\int_{0}^{t} \int_{N}^{\infty} G_{\lambda}(t-s, x, y) f(u) d y d s-\int_{0}^{t} \int_{-\infty}^{N} G_{\lambda_{N}}(t-s, x, y) f(u) d y d s .
\end{aligned}
$$

As $N \rightarrow \infty$ the limit of each term on the right hand side of (8) can be shown to be 0 . The limit for the first term follows from Theorem 2. The second term requires an estimate identical to Lemma 6. Finally the last two terms can be shown to have limit 0 by the methods of Lemmas 4 and 5 .

LEMma 8. $u$ is the unique solution of (2) in $B_{k}$.

Proof. Let $v$ be another solution of (2). Then

$$
v=-\int_{0}^{t} \int_{-\infty}^{+\infty} G_{\lambda}(t-s, x, y) f(v) d y d s+W(x, t) .
$$

Hence

$$
u-v=-\int_{0}^{t} \int_{-\infty}^{+\infty} G_{\lambda}(t-s, x, y)(f(u)-f(v)) d y d s
$$

and

$$
\begin{aligned}
\int_{0}^{T} \int_{-}^{+} \exp (-k|x|)(f(u)-f(v))(u-v) d x d t \\
=\int_{0}^{T} \int_{-\infty}^{+\infty} \exp (-k|x|)(f(u)-f(v)) \\
\quad \times\left(-\int_{0}^{t} \int_{-\infty}^{+\infty} G_{\lambda}(t-s, x, y)(f(u)-f(v)) d y d s\right) d x d t
\end{aligned}
$$


Using estimates similar to those in the proof of Lemmas 2 and 3 it follows that $|u-v|_{k}^{p} \leqq 0$ or $u=v$.

In order to investigate the stationary distribution of $u$ as $t \rightarrow \infty$ a sequence of approximations $\hat{u}_{N}$ must be constructed. Let $W_{N}(x, t)$ be the Gaussian processes with mean 0 and covariance

$$
\begin{aligned}
& E\left(W_{N}(x, t) W_{M I}(y, s)\right) \\
& \quad=\int_{0}^{\min (t, s)} \int_{-N}^{+N} G_{\lambda N}(t-r, x, z) G_{\lambda M}(s-r, y, z) d z d r(M \geqq N) .
\end{aligned}
$$

Also

$$
\begin{aligned}
& E\left(W_{N}(x, t) W(y, s)\right) \\
& \quad=\int_{0}^{\min (t, s)} \int_{-N}^{+N} G_{\lambda N}(t-r, x, z) G_{\lambda}(s-r, y, z) d z d r .
\end{aligned}
$$

Formally

$$
W_{N}(x, t)=\int_{0}^{t} \int_{-N}^{+N} G_{\lambda_{N}}(t-s, x, y) \alpha(y, s) d y d s .
$$

Note $\lim _{N \rightarrow \infty}\left|W_{N}(x, t)-W(x, t)\right|_{k}=0$ almost surely follows from the convergence of covariance for Gaussian processes.

Lemma 9. The equation

$$
\widehat{u}_{N}=-\int_{0}^{t} \int_{-N}^{+N} G_{2 N}(t-s, x, y) f\left(\hat{u}_{N}\right) d y d s+W_{N}(x, t)
$$

has a unique solution with $\left|\hat{u}_{N}\right|_{o k}<c$.

Proof. The proof is identical to Theorem 1.

LEMMA 10. $\lim _{N \rightarrow \infty}\left|u_{N}-\widehat{u}_{N}\right|_{p}=0$ almost surely. Hence $\lim _{N \rightarrow \infty} \widehat{u}_{N}=u$ almost surely.

Proof.

$$
u_{N}-\widehat{u}_{N}=\int_{0}^{t} \int_{-N}^{+N} G_{\lambda}(t-s, x, y)\left(f\left(u_{N}\right)-f\left(\widehat{u}_{N}\right)\right) d y d s+W(x, t)-W_{N}(x, t) .
$$

Then it follows

$$
\begin{aligned}
& \int_{0}^{T} \int_{-\infty}^{+\infty} \exp (-k|x|)\left(f\left(u_{N}\right)-f\left(\hat{u}_{N}\right)\right)\left(u_{N}-\widehat{u}_{N}\right) d x d t \\
& =\int_{0}^{T} \int_{-\infty}^{+\infty} \exp (-k|x|)\left(f\left(u_{N}\right)-f\left(\hat{u}_{N}\right)\right) \\
& \quad \quad \times \int_{0}^{t} \int_{-N}^{+N} G_{\lambda}(t-s, x, y)\left(f\left(u_{N}\right)-f\left(\hat{u}_{N}\right)\right) d y d s \\
& \quad+\int_{0}^{T} \int_{-\infty}^{+\infty} \exp (-k|x|)\left(f\left(u_{N}\right)-f\left(\hat{u}_{N}\right)\right)\left(W(x, t)-W_{N}(x, t)\right) d x d t .
\end{aligned}
$$


Once again using the methods of Lemma 2 and Lemma 3 it follows that

$$
\begin{aligned}
& \lim _{N \rightarrow \infty} c\left|u_{N}-\hat{u}_{N}\right|^{p} \\
& \quad \leqq \lim _{N \rightarrow \infty} \int_{0}^{T} \int_{-\infty}^{+\infty} \exp (-k|x|)\left(f\left(u_{N}\right)-f\left(\hat{u}_{N}\right)\right)\left(W(x, t)-W_{N}(x, t)\right) d x d t=0
\end{aligned}
$$

almost surely which implies $\lim _{N \rightarrow \infty} \hat{u}_{N}=\lim _{N \rightarrow \infty} u_{N}=u$ completing the proof.

Using the methods of Lemma 2 through Lemma 9 of Marcus [2] it can be shown that $\hat{u}_{N}(x, t)=R_{N}(x, t)+V_{N}(x, t)$ where $R_{N}$ is a stationary process on $B_{k}$ and $V_{N}$ satisfies

$$
E\left(\int_{-\infty}^{+\infty} V_{N}^{2}(x, t) \exp (-k|x|) d x\right) \leqq c_{1} \exp \left(-c_{2} t\right)
$$

where $c_{1}, c_{2}>0$ are independent of $N$.

LEMMA 11. $R \equiv \lim _{N \rightarrow \infty} R_{N}$ exists in $B_{k}$ and is a stationary process on $L_{k}^{p}$.

Proof. The proof is identical to that of Lemma 8 of Marcus [2]. Define $V \equiv u-R$.

LEMMA 12.

$$
\lim _{t \rightarrow \infty} E\left(\int_{-\infty}^{+\infty} \exp (-k|x|) V^{2}(x, t) d x\right)=0 .
$$

Proof. The proof is identical to Lemma 9 of Marcus [2].

THEOREM 3. The unique solution of

$$
u(x, t)=-\int_{0}^{t} \int_{-\infty}^{+\infty} G_{\lambda}(t-s, x, y) f(u(y, s)) d s+W(x, t)
$$

can be represented as $u(x, t)=R(x, t)+V(x, t)$ where $R(x, t)$ is a stationary process on $L_{k}^{p}$ and

$$
\lim _{t \rightarrow \infty} E\left(\int_{-\infty}^{+\infty} \exp (-k|x|) V^{2}(x, t) d x\right)=0 .
$$

Proof. This follows immediately from Lemmas 10,11, and 12.

The next problem is to obtain information about the stationary distribution of $R$ using $R_{N}$. Let $F(u) \equiv \int_{0}^{u} f(v) d v$. Note $0 \leqq F(u)<$ $c\left(|u|^{p}+|u|\right)$ follows easily from properties (i) and (ii) of $f$. 
LEMMA 13. The stationary distributions of $R_{N}$ have a RadonNikodym derivative proportional to $\exp \left(-\int_{-N}^{+N} F\left(u_{o N}\right) d x\right)$ with respect to the Gaussian measure on $u_{0_{N}} \in L_{k}^{p}$ with mean 0 and covariance $\int_{0}^{\infty} G_{\lambda_{N}}(2 s, x, y) d s$.

Proof. This result is proved in Lemma 10 of Marcus [2].

LEMmA 14. Let $u_{0} \in L_{k}^{p}$ be a Gaussian random variable with mean 0 and convariance

$$
E\left(u_{0}(x) u_{0}(y)\right)=\int_{0}^{\infty} G_{\lambda}(2 t, x, y) d t=\frac{1}{2} \exp (-\lambda|x-y|) .
$$

Let $g$ be a bounded continuous function on $L_{k}^{p}$. Then

$$
\begin{aligned}
E(g(R))= & \lim _{t \rightarrow \infty} E(g(u))=\lim _{N \rightarrow \infty} E\left(g\left(u_{0}\right) \exp \left(-\int_{-N}^{+N} F\left(u_{0}\right) d x\right)\right) \\
& / E\left(\exp \left(-\int_{-N}^{+N} F\left(u_{0}\right) d x\right)\right) .
\end{aligned}
$$

(Note that the covariance of $u_{0}(x)$ is equal to $\lim _{t \rightarrow \infty} E(W(x, t) W(y, t))=$ $\left.\lim _{t \rightarrow \infty} \lim _{N \rightarrow \infty} E\left(W_{N}(x, t) W_{N}(y, t)\right)=\lim _{N \rightarrow \infty} E\left(u_{O N}(x) u_{o N}(y)\right).\right)$

Proof. Since $\lim _{N \rightarrow \infty} R_{N}=R$ and $\lim _{t \rightarrow \infty}|u-R|=0$ in mean square, it follows from the bounded convergence theorem that

$$
\begin{gathered}
E(g(R))=\lim _{t \rightarrow \infty} E(g(u))=\lim _{N \rightarrow \infty} E\left(g\left(u_{O N}\right) \exp \left(-\int_{-N}^{+N} F\left(u_{O N}\right) d x\right)\right) \\
/ E\left(\exp \left(-\int_{-N}^{+N} F\left(u_{O N}\right) d x\right)\right)=\lim _{N \rightarrow \infty} E\left(g\left(R_{N}\right)\right) .
\end{gathered}
$$

Since as random processes on $[-N,+N]$ and also in $L_{k}^{p}, u_{O N}$ converge weakly to $u_{0}$ then by bounded convergence using the growth condition on $F$ it is possible to show that (see [1])

$$
\begin{array}{r}
\lim _{N \rightarrow \infty} E\left(g\left(u_{O N}\right) \exp \left(-\int_{-N}^{+N} F\left(u_{O N}\right) d x\right)\right) / E\left(\exp \left(-\int_{-N}^{+N} F\left(u_{O N}\right) d x\right)\right) \\
=\lim _{N \rightarrow \infty} E\left(g\left(u_{0}\right) \exp \left(-\int_{-N}^{+N} F\left(u_{0}\right) d x\right)\right) / E\left(\exp \left(-\int_{-N}^{+N} F\left(u_{0}\right) d x\right)\right)
\end{array}
$$

which completes the proof.

In conclusion it is interesting to note that the stationary distribution of $R$ is never absolutely continuous with respect to the stationary distribution of $W(x, t)$ since $\lim _{N \rightarrow \infty} E\left(\exp \left(-\int_{-N}^{+N} F\left(u_{0}\right) d x\right)\right)=0$. 
APPENDIX. Let $f(u)=u^{3}$. Then conditions (i) and (ii) are satisfied with $p=4$. The results of this paper can then be applied to the equation:

$$
\begin{aligned}
\frac{\partial u}{\partial t}(x, t)= & \frac{1}{2} \frac{\partial^{2} u}{\partial x^{2}}(x, t)-\lambda^{2} u(x, t)-u^{3}(x, t)+\alpha(x, t) \\
& (-\infty<x<\infty \text { and } u(x, 0)=0)
\end{aligned}
$$

$\alpha(x, t)$ is a "white noise" process i.e., a generalized Gaussian random process satisfying formally $E(\alpha(x, t))=0$ and $E(\alpha(x, t) \alpha(y, s))=$ $\delta(x-y) \delta(t-s)$.

From Theorem 2, Lemma 7 and Lemma 8, equation (9) has a unique generalized solution $u(x, t)$ satisfying

$$
\int_{0}^{T} \int_{-\infty}^{+\infty} \exp (-k|x|) u^{4}(x, t) d x d t<\infty
$$

almost surely for some $k>0$. From Theorem 3 and Lemmas 10, 11, $12 u(x, t)=R(x, t)+V(x, t)$ where $R(x, t)$ is a stationary process in $t$ and $\lim _{t \rightarrow \infty} E\left(\int_{-\infty}^{+\infty} \exp (-k|x|) V^{2}(x, t) d x\right)=0$. From Lemmas 13 and 14 if $g$ is a bounded continuous function then $\lim _{t \rightarrow \infty} E(g(u))=$ $E(g(R))$

$$
=\lim _{N \rightarrow \infty} E\left(g\left(u_{0}\right) \exp \left(-\int_{-N}^{+N} u_{0}^{4}(x) d x\right)\right) / E\left(\exp \left(-\int_{-N}^{+N} u_{0}^{4}(x) d x\right)\right)
$$

where $u_{0}$ is a Gaussian process on the real line with expectation 0 and covariance $E\left(u_{0}(x) u_{0}(y)\right)=\exp (-\lambda|x-y|)$. The stationary distribution of $R$ corresponds to the measure associated with $\left(\phi^{4}\right)_{1}$ in constructive quantum field theory. See Rosen and Simon [4].

\section{REFERENCES}

1. Guerra, Rosen, and Simon, The $P(\phi)_{2}$ Euclidean quantum field theory as classical statistical mechanics, Annals of Math., 101 (1975), 111-259.

2. Marcus, Parabolic Ito equations with monotone non-linearities, Functional Analysis, 29 (1978), 275-286.

3. Nelson, Construction of quantum fields from Markov fields, Functional Analysis, 12 (1973), 97-112.

4. Rosen and Simon, Fluctuations in $P(\phi)$, processes, Annals of Probability, 4 (1976), 155-174.

5. Vainberg, Variational Method and Method of Monotone Operators in the Theory of Nonlinear Equations, Wiley, 1973.

Received May 9, 1978.

College of Staten Island

715 OCEAN TERRACE

STATEN ISLAND, NY 10301 



\section{PACIFIC JOURNAL OF MATHEMATICS}

\section{EDITORS}

DONALD BABBITT (Managing Editor)

University of California

Los Angeles, California 90024

Hugo RossI

University of Utah

Salt Lake City, UT 84112

C. C. MOORE and ANDrew OGG

University of California

Berkeley, CA 94720
J. DUgundjI

Department of Mathematics University of Southern California Los Angeles, California 90007

R. FinN aNd J. Milgram Stanford University Stanford, California 94305

\section{ASSOCIATE EDITORS}

E. F. BECKENBACH

B. H. NeUMANN

F. WOLF

K. YosHIDA

\section{SUPPORTING INSTITUTIONS}

UNIVERSITY OF BRITISH COLUMBIA CALIFORNIA INSTITUTE OF TECHNOLOGY UNIVERSITY OF CALIFORNIA MONTANA STATE UNIVERSITY UNIVERSITY OF NEVADA, RENO NEW MEXICO STATE UNIVERSITY OREGON STATE UNIVERSITY UNIVERSITY OF OREGON
UNIVERSITY OF SOUTHERN CALIFORNIA STANFORD UNIVERSITY UNIVERSITY OF HAWAII UNIVERSITY OF TOKYO UNIVERSITY OF UTAH WASHINGTON STATE UNIVERSITY UNIVERSITY OF WASHINGTON 


\section{Pacific Journal of Mathematics}

\section{Vol. 84 , No. 1 \\ May, 1979}

Michael James Beeson, Goodman's theorem and beyond ...............

Robert S. Cahn and Michael E. Taylor, Asymptotic behavior of multiplicities

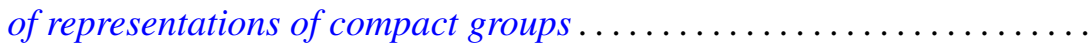

Douglas Michael Campbell and Vikramaditya Singh, Valence properties of

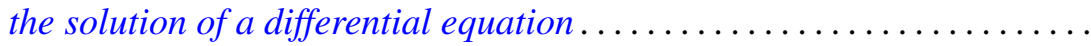

J.-F. Colombeau, Reinhold Meise and Bernard Perrot, A density result in spaces of Silva holomorphic mappings .....................

Marcel Erné, On the relativization of chain topologies .................

Le Baron O. Ferguson, Uniform and $L_{p}$ approximation for generalized

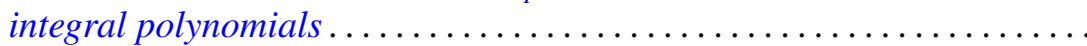

Kenneth R. Goodearl and David E. Handelman, Homogenization of regular rings of bounded index..................................

Friedrich Haslinger, A dual relationship between generalized

Abel-Gončarov bases and certain Pincherle bases .................

Miriam Hausman, Generalization of a theorem of Landau. .

Makoto Hayashi, 2-factorization in finite groups

Robert Marcus, Stochastic diffusion on an unbounded domain ........... 143

Isabel Dotti de Miatello, Extension of actions on Stiefel manifolds....

C. David (Carl) Minda, The hyperbolic metric and coverings of Riemann surfaces...

Somashekhar Amrith Naimpally and Mohan Lal Tikoo, On $T_{1}$-compactifications.

Chia-Ven Pao, Asymptotic stability and nonexistence of global solution for a semilinear parabolic equation ....

Shigeo Segawa, Harmonic majoration of quasibounded type ...

Sze-Kai Tsui and Steve Wright, The splitting of operator algebras ...

Bruce Williams, Hopf invariants, localization and embeddings of Poincaré complexes....

Leslie Wilson, Nonopenness of the set of Thom-Boardman maps ...

Alicia B. Winslow, There are $2^{\mathrm{c}}$ nonhomeomorphic continua in $\beta R^{n}-R^{n}$ 